\title{
ASYMPTOTIC-PRESERVING METHODS FOR HYPERBOLIC AND TRANSPORT EQUATIONS WITH RANDOM INPUTS AND DIFFUSIVE SCALINGS
}

\author{
SHI JIN*, DONGBIN XIU ${ }^{\dagger}$, AND XUEYU ZHU
}

\begin{abstract}
In this paper we develop a set of stochastic numerical schemes for hyperbolic and transport equations with diffusive scalings and subject to random inputs. The schemes are asymptotic preserving (AP), in the sense that they preserve the diffusive limits of the equations in discrete setting, without requiring excessive refinement of the discretization. Our stochastic AP schemes are extensions of the well-developed deterministic AP schemes. To handle the random inputs, we employ generalized polynomial chaos (gPC) expansion and combine it with stochastic Galerkin procedure. We apply the gPC Galerkin scheme to a set of representative hyperbolic and transport equations and establish the AP property in the stochastic setting. We then provide several numerical examples to illustrate the accuracy and effectiveness of the stochastic AP schemes.
\end{abstract}

Key words. Uncertainty quantification, hyperbolic systems, transport equations, diffusion limit, asympotic-preserving, generalized polynomial chaos

1. Introduction. Many kinetic and hyperbolic equations often have diffusive scaling that asymptotically leads to diffusion equations. When the equations are near the diffusive regime, numerical simulations become prohibitively expensive, due to the exceedingly small parameters, e.g., the particle mean free path, relaxation time, etc., that need to be numerically resolved. Asymptotic-preserving (AP) schemes are those that mimic the asymptotic transitions from the kinetic or hyperbolic equations to their diffusive limits in discrete setting $[25,24,19,15]$. Since the mid-1990's, the development of AP schemes for such problems has generated many interests, see, for example, $[20,16,17,11,22,12,26]$. The AP strategy has been proved to be a powerful and robust technique to address multiscale problems in many kinetic and hyperbolic problems. The main advantage of AP schemes is that they are very efficient in the diffusive regime, for they do not need to resolve the small diffusive parameters numerically and yet can still capture the macroscopic behavior governed by the limiting diffusion equations. For example, it was proved, in the case of linear transport with a diffusive scaling, the AP scheme converges uniformly with respect to the scaling parameter ([11]). This is expected to be true for all AP schemes [15], although specific proofs are needed for specific problems. For many multi-scale problems, carefully constructed AP schemes avoid the difficulty of coupling a microscopic solver with a macroscopic one, as the micro solver automatically becomes a macro solver in the diffusive regime.

In practical applications, kinetic and hyperbolic problems almost always contain parameters that are uncertain, due to modeling and/or experimental errors. In this paper, we investigate numerical methods for such problems and aim to develop stochastic version of the AP schemes that can quantify the uncertainty in the diffusive regime. While well established for the deterministic kinetic or hyperbolic equations, AP schemes have not been constructed for hyperbolic and kinetic problems with ran-

*Department of Mathematics, Institute of Natural Sciencces and MOE-LSC, Shanghai Jiao Tong University, Shanghai 200240, China; and Department of Mathematics, University of Wisconsin, Madision, WI 53706, USA. Email: jin@math.wisc.edu

${ }^{\dagger}$ Scientific Computing and Imagining Institute and Department of Mathematics, University of Utah, Salt Lake City, UT 84112, USA. Email: dongbin.xiu@utah.edu

${ }^{\ddagger}$ Scientific Computing and Imaging Institute, University of Utah, Salt Lake City, UT 84112, USA. Email: xzhu@sci.utah.edu. 
dom inputs. The few existing work on uncertainty quantification of hyperbolic and transport equations $([13,8,14,6,30])$ focused on efficient methods in the standard stochastic setting and did not study the asymptotic behavior of these methods. Consequently, those methods do not possess AP property.

In this paper, we present a set of stochastic AP methods for kinetic and hyperbolic problems with random inputs. To cope with the random inputs, we employ generalized polynomial chaos (gPC) approach [35], combined with stochastic Galerkin (SG) method. While the gPC-SG approach has been adopted for a large variety of stochastic problems, this paper represents the first attempt, to our best knowledge, to construct stochastic AP (sAP) schemes. The sAP methods are constructed by extending the idea from the deterministic AP methods ([16, 17]), and are illustrated via three prototype kinetic equations: the linear Goldstein-Taylor model, the nonlinear Carleman model, and the linear transport equation. The selected equations are representative, as they possess features such as linearity vs. nonlinearity, representations in physical space $v s$. in phase space. We will demonstrate that the carefully constructed gPC-Galerkin method can be AP, in the sense that in the diffusive regime it automatically becomes a gPC-Galerkin approximation for the limiting stochastic diffusion equations. This holds true for fixed time step, mesh size and order of the gPC expansions, and without the requirement for refinement.

It is interesting to note that one obvious approach to extend the deterministic AP methods to random domain is to adopt stochastic collocation (SC) approach. SC methods are based on sampling and can be easily applied to any systems with established deterministic solvers. One can apply a deterministic AP scheme to each individual samples, solve them separately to obtain the solution ensemble, and then construct an approximation, e.g., a gPC approximation, to the stochastic solution in the randoms space. However, it is important to note that even though each samples satisfy the AP properties, there is no guarantee that the global approximation is AP in the entire random space. This is especially true in higher dimensional random spaces, as the errors incurred by the construction (e.g., interpolation errors) can be highly non-trivial away from the samples. On the other hand, the proposed sAP is based on the Galerkin formulation, and can be proven to be AP throughout the random domain, for any fixed (and even low) order of gPC expansions. (For reviews of the basic properties of SC methods, the interested readers are referred to [33].)

The rest of the paper is organized as follows: in the next section we discuss some preliminary materials and give a formal definition of stochastic AP. In Section 3 we shall briefly review the three model problems under consideration and their diffusive limits. We then present the details of the stochastic AP schemes for these equations and establish their AP properties in next three sections. After a discussion on the use of stochastic collocation methods in Section 7 , we present several numerical examples to illustrate the effectiveness of the sAP methods in Section 8.

2. Preliminaries. Let us consider a class of problems, e.g., kinetic and hyperbolic problems, with diffusive scaling. Without being specific, we illustrate the idea of asymptotic-preserving (AP) scheme in the following manner. Consider a system

$$
\partial_{t} u^{\epsilon}=\mathcal{L}^{\epsilon}\left(t, x, u^{\epsilon} ; \epsilon\right),
$$

where $\epsilon$ is the scaling parameter. Proper initial and boundary conditions are assumed. As $\epsilon \rightarrow 0$, the diffusive limit satisfies

$$
\partial_{t} u=\mathcal{L}(t, x, u)
$$


Let $v_{h}^{\epsilon}$ be a numerical solution to (2.1), where the subscript $h$ denotes the discretization in spatial and temporal domains. Obviously, $v_{h}^{\epsilon}$ is obtained via a proper numerical scheme. The scheme is called AP if its limiting solution $v_{h}=\lim _{\epsilon \rightarrow 0} v_{h}^{\epsilon}$ solves (2.2) automatically, without refining the discretization $h$. Such kind of AP schemes are well studied for deterministic kinetic and hyperbolic problems, see, for example, [20, 16, 17, 11, 22, 12, 26].

2.1. Stochastic asymptotic preserving scheme. We now consider the same problem subject to random inputs.

$$
\partial_{t} u^{\epsilon}=\mathcal{L}^{\epsilon}\left(t, x, z, u^{\epsilon} ; \epsilon\right),
$$

where $z \in I_{z} \subseteq \mathbb{R}^{d}, d \geq 1$, are a set of random variables equipped with probability density function $\rho$. These random variables characterize the random inputs into the system. As $\epsilon \rightarrow 0$, the diffusive limit becomes

$$
\partial_{t} u=\mathcal{L}(t, x, z, u) .
$$

We now extend the concept of deterministic AP to the stochastic case. To avoid the cluttering of notations, let us now focus on the discretization in the random space $I_{z}$.

Definition 2.1 (Stochastic AP). Let $\mathcal{S}$ be a numerical scheme for (2.3), which results in a solution $v^{\epsilon}(z) \in V_{z}$ in a finite dimensional linear function space $V_{z}$. Let $v(z)=\lim _{\epsilon \rightarrow 0} v^{\epsilon}(z)$ be its asymptotic limit. We say that the scheme $\mathcal{S}$ is strongly asymptotic perserving if the limiting solution $v(z)$ satisfies the limiting equation (2.4) for almost every $z \in I_{z}$; and it is weakly asymptotic perserving if the limiting solution $v(z)$ satisfies the limiting equation (2.4) in a weak form.

The strong AP is obviously very strong to acquire, as it requires $v(z)$ to be (almost) the analytical solution of (2.4) in the random space. On the other hand, the weak AP is more amenable and practical. In the finite dimensional linear space $V_{z}$, it can be acquired via, for example, a Galerkin formulation by enforcing the residue $v_{t}-\mathcal{L}(t, x, z, v)$ to be orthogonal to $V_{z}$. Hereafter we will only discuss the weak AP solution and commonly refer it as stochastic asymptotic preserving (sAP). Note that, similar to the deterministic case, the limiting sAP solution $v(z)$ becomes a weak solution to the limiting equation (2.4) automatically, for any given space $V_{z}$ and without the need to enrich it.

2.2. Generalized polynomial chaos and stochastic Galerkin. We now briefly review the gPC method $([9,35])$ and its Galerkin formulation for stochastic PDEs, particularly for the random diffusion equation

$$
\partial_{t} u=\partial_{x}\left[a(x, z) \partial_{x} u\right],
$$

where $0<a_{\min } \leq a(x, z) \leq a_{\max }<+\infty$ for all $x, z$.

In the most common gPC setting, one seeks a numerical solution in term of $d$ variate orthogonal polynomials of degree $N \geq 1$. That is, the linear space $V_{z}$ is set to be $\mathbb{P}_{N}^{d}$, the space of $d$-variate orthogonal polynomials of degree up to $N \geq 1$. For random variable $z \in \mathbb{R}^{d}$, one then seeks

$$
u(x, t, z) \approx u_{N}(x, t, z)=\sum_{m=1}^{M} \hat{u}_{m}(t, x) \Phi_{m}(z), \quad M=\left(\begin{array}{c}
d+N \\
d
\end{array}\right),
$$

where $\left\{\Phi_{m}(z)\right\} \subset \mathbb{P}_{N}^{d}$ are orthonormal polynomials satisfying

$$
\int \Phi_{i}(z) \Phi_{j}(z) \rho(z) d z=\delta_{i j}, \quad 1 \leq i, j \leq M=\operatorname{dim}\left(\mathbb{P}_{N}^{d}\right) .
$$


Here $\rho(z)$ is the probability density function of $z$ and $\delta_{i j}$ the Kronecker delta function. Note that when the random dimension $d>1$, an ordering scheme for multiple index is required to re-order the polynomials into a single index $m$ here. Typically, the graded lexicographic order is used, see, for example, Section 5.2 of [33]. The random dimensionality $d$ is determined by the number of random variables $z$ used in the input $a(x, z)$, which is typically modeled by a linear combination of $z$, i.e.,

$$
a(x, z) \approx \sum_{i=1}^{d} \hat{a}_{i}(x) z_{i} .
$$

The most widely used model is the Karhunen-Loeve expansion. (See, for example, $[9,33]$ for general discussions.)

In the gPC Galerkin formulation, one inserts the approximation $u_{N}(2.6)$ into the governing equation and then enforces the residue to be orthogonal to $\mathbb{P}_{N}^{d}$. Upon doing so, one obtains a set of deterministic equations for the expansion coefficients $\left\{\hat{u}_{m}\right\}$

$$
\partial_{t} \hat{\mathbf{u}}(x, t)=\partial_{x}\left(\mathbf{A}(x) \partial_{x} \hat{\mathbf{u}}\right),
$$

where

$$
\hat{\mathbf{u}}=\left(\hat{u}_{1}, \cdots, \hat{u}_{M}\right)^{T},
$$

is the coefficient vector and $\mathbf{A}=\left(a_{i j}\right)_{1 \leq i, j \leq M}$ with

$$
a_{i j}(x)=\int a(x, z) \Phi_{i}(z) \Phi_{j}(z) \rho(z) d z .
$$

Obviously, $\mathbf{A}(x)$ is real and symmetric. It is also straightforward to show that the eigenvalues of $\mathbf{A}$ are bounded by the range of $a$.

Lemma 2.2. For any $x$, let $\lambda_{1}(x) \geq \lambda_{2}(x) \geq \cdots$ be the eigenvalues of $\mathbf{A}(x)$, defined by (2.10), and $a_{\min } \leq a(x, z) \leq a_{\max }$ for all $x$ and $z$. Then,

$$
a_{\min } \leq \lambda_{i}(x) \leq a_{\max }, \quad \forall i .
$$

Proof. The proof is a trivial extension of the Theorem 2.1 of [13], by considering $a(x, z)-a_{\min }$ and $a(x, z)-a_{\max } . \square$

In the random diffusion equation (2.5), the assumption of $0<a_{\min } \leq a(x, z) \leq$ $a_{\max }<+\infty$ for all $x, z$ immediately leads to the conclusion that $\mathbf{A}$ is positive definite ([36]).

3. Three kinetic or hyperbolic equations. In this section we present three representative kinetic equations subject to random inputs and discuss their diffusion limits.

3.1. The random Goldstein-Taylor model. We first consider a one dimensional random Goldstein-Taylor model under diffusive scaling:

$$
\left\{\begin{array}{l}
\partial_{t} u+\partial_{x} v=0, \\
\partial_{t} v+\frac{1}{\epsilon} a(x, z) \partial_{x} u=-\frac{1}{\epsilon} v .
\end{array}\right.
$$

In the deterministic Goldstein-Taylor model [10,31], the wave speed $a(x, z) \equiv 1$. Here we assume a random wave speed, a case that has many interesting applications on 
its own right [13]. In the diffusion limit $\epsilon \rightarrow 0^{+},(3.1)$ can be approximated to the leading order by the following heat equation with random diffusion coefficient $a(x, z)$ :

$$
\left\{\begin{array}{l}
v=-a(x, z) \partial_{x} u, \\
\partial_{t} u=\partial_{x}\left(a(x, z) \partial_{x} u\right) .
\end{array}\right.
$$

3.2. The random Carleman model. The second example is the one-dimensional nonlinear random Carleman model under the diffusive scaling:

$$
\left\{\begin{array}{l}
\partial_{t} u+\partial_{x} v=0 \\
\partial_{t} v+\frac{1}{\epsilon} \partial_{x} u=-\frac{1}{\epsilon} \kappa(x, z) u v .
\end{array}\right.
$$

In the deterministic Carleman model $([3]), \kappa(x, z) \equiv 1$. In general, the randomness in the source term of a hyperbolic balance law could arise from friction, topography or random medium. Indeed, certain kinetic equations have been developed for porous media $([27,29])$, where $u$ on the right side of (3.3) is replaced by $u^{\beta}$ with $\beta \leq-1$, to model the uncertainty in the media $([5])$. The corresponding limiting nonlinear diffusion equation, as $\epsilon \rightarrow 0^{+}$, is

$$
\left\{\begin{array}{l}
v=-\frac{1}{\kappa(x, z) u} \partial_{x} u \\
\partial_{t} u=\partial_{x}\left[\frac{1}{\kappa(x, z) u} \partial_{x} u\right] .
\end{array}\right.
$$

3.3. The linear transport equation. Finally, we consider a one-dimensional linear transport equation with random scattering coefficients under the diffusive scaling. Let $f(t, x, v)$ be the probability density distribution of particles at position $x$, time $t$, and with $v \in(-1,1)$ the cosine of the angle between the particle velocity and its position variable. Then, $f$ is governed by the following linear transport equation $([4])$ :

$$
\epsilon \partial_{t} f+v \partial_{x} f=\frac{\sigma(x, z)}{\epsilon}\left[\frac{1}{2} \int_{-1}^{1} f\left(v^{\prime}\right) d v^{\prime}-f\right],
$$

where $\sigma(x, z)$ is the random scattering coefficient. In this equation we ignored the absorption and source terms, which can also be random. The treatment of these terms does not add further numerical difficulties and will be neglected here for the ease of presentation.

To understand its diffusion limit, we first split this equation into two equations for $v>0$ :

$$
\begin{aligned}
& \epsilon \partial_{t} f(v)+v \partial_{x} f(v)=\frac{\sigma(x, z)}{\epsilon}\left[\frac{1}{2} \int_{-1}^{1} f\left(v^{\prime}\right) d v^{\prime}-f(v)\right] \\
& \epsilon \partial_{t} f(-v)-v \partial_{x} f(-v)=\frac{\sigma(x, z)}{\epsilon}\left[\frac{1}{2} \int_{-1}^{1} f\left(v^{\prime}\right) d v-f(-v)\right],
\end{aligned}
$$

and then consider its even and odd parities

$$
\begin{aligned}
& r(t, x, v)=\frac{1}{2}[f(t, x, v)+f(t, x,-v)], \\
& j(t, x, v)=\frac{1}{2 \epsilon}[f(t, x, v)-f(t, x,-v)] .
\end{aligned}
$$


The system (3.6) can then be rewritten as follows:

$$
\left\{\begin{array}{l}
\partial_{t} r+v \partial_{x} j=\frac{\sigma(x, z)}{\epsilon^{2}}(\bar{r}-r), \\
\partial_{t} j+\frac{v}{\epsilon^{2}} \partial_{x} r=-\frac{\sigma(x, z)}{\epsilon^{2}} j .
\end{array}\right.
$$

where

$$
\bar{r}(t, x)=\int_{0}^{1} r d v
$$

As $\epsilon \rightarrow 0^{+},(3.8)$ yields

$$
r=\bar{r}, \quad j=-\frac{v}{\sigma(x, z)} \partial_{x} \bar{r} .
$$

Substituting this into system (3.8) and integrating over $v$, one gets the limiting diffusion equation $([23,1])$ :

$$
\partial_{t} \bar{r}=\partial_{x}\left[\frac{1}{3 \sigma(x, z)} \partial_{x} \bar{r}\right]
$$

4. Stochastic AP scheme for the random Goldstein-Taylor model. We now derive a stochastic AP scheme for the random Goldstein-Taylor model (3.1). The scheme is based on the gPC Galerkin formulation, where again we seek polynomial approximations to the solutions, in the form of $(2.6)$,

$$
u_{N}(x, z, t)=\sum_{m=1}^{M} \hat{u}_{m}(t, x) \Phi_{m}(z), \quad v_{N}(x, z, t)=\sum_{m=1}^{M} \hat{v}_{m}(t, x) \Phi_{m}(z) .
$$

Let

$$
\hat{\mathbf{u}}=\left(\hat{u}_{1}, \cdots, \hat{u}_{M}\right)^{T}, \quad \hat{\mathbf{v}}=\left(\hat{v}_{1}, \cdots, \hat{v}_{M}\right)^{T},
$$

be the coefficient vectors and conduct the Galerkin projection on the governing equation, we obtain

$$
\left\{\begin{array}{l}
\partial_{t} \hat{\mathbf{u}}+\partial_{x} \hat{\mathbf{v}}=0 \\
\partial_{t} \hat{\mathbf{v}}+\frac{1}{\epsilon} \mathbf{A}(x) \partial_{x} \hat{\mathbf{u}}=-\frac{1}{\epsilon} \hat{\mathbf{v}}
\end{array}\right.
$$

where $\mathbf{A}=\left(a_{i j}\right)_{1 \leq i, j \leq M}$ is defined in (2.10).

4.1. AP time splitting. We now extend the deterministic AP time splitting introduced in [16] to the gPC Galerkin system (4.2). The procedure starts with a reformulation of (4.2) as a (nonstiff) linear hyperbolic equation system with a stiff relaxation term as follows:

$$
\left\{\begin{array}{l}
\partial_{t} \hat{\mathbf{u}}+\partial_{x} \hat{\mathbf{v}}=0 \\
\partial_{t} \hat{\mathbf{v}}+\alpha \partial_{x} \hat{\mathbf{u}}=-\frac{1}{\epsilon}\left[\hat{\mathbf{v}}+\left(\mathbf{A}-\epsilon \alpha \mathbf{I}_{M}\right) \partial_{x} \hat{\mathbf{u}}\right]
\end{array}\right.
$$

where $\mathbf{I}_{M}$ is the $M \times M$ identity matrix, and $\alpha>0$ is a real parameter, chosen such that $\left(\mathbf{A}-\epsilon \alpha \mathbf{I}_{M}\right)$ remains positive definite for any $\epsilon([21,19])$. Without loss of 
generality, we shall assume $\epsilon \ll 1$. Since the eigenvalues of $\mathbf{A}(x)$ are bounded by the range of $a(x, z)$ (see Lemma 2.2), then it suffices to choose $\alpha<a_{\min }$.

The deterministic AP time-splitting method introduced in [16] can now be applied to (4.3). It consists of the following two steps:

- Relaxation step:

$$
\left\{\begin{array}{l}
\partial_{t} \hat{\mathbf{u}}=0 \\
\partial_{t} \hat{\mathbf{v}}=-\frac{1}{\epsilon}\left[\hat{\mathbf{v}}+\left(\mathbf{A}-\epsilon \alpha \mathbf{I}_{M}\right) \partial_{x} \hat{\mathbf{u}}\right] .
\end{array}\right.
$$

- Convection step:

$$
\left\{\begin{array}{l}
\partial_{t} \hat{\mathbf{u}}+\partial_{x} \hat{\mathbf{v}}=0 \\
\partial_{t} \hat{\mathbf{v}}+\alpha \partial_{x} \hat{\mathbf{u}}=0 .
\end{array}\right.
$$

As $\epsilon \rightarrow 0^{+}$, the relaxation step yields

$$
\hat{\mathbf{v}}=-\mathbf{A}(x) \partial_{x} \hat{\mathbf{u}}
$$

which, when substituted into the convection step, gives

$$
\partial_{t} \hat{\mathbf{u}}=\partial_{x}\left(\mathbf{A}(x) \partial_{x} \hat{\mathbf{u}}\right) .
$$

This is exactly the gPC Galerkin scheme (2.9) for the diffusion equation (3.2). By Definition 2.1, the time splitting method presented here is sAP (in the weak form).

4.2. AP for the fully discretized system. We now adopt a finite volume method to discuss the sAP property of the time-splitting scheme in the fully discretized form. For spatial discretization, we choose the spatial grids $x_{i+1 / 2}$ with a uniform mesh size $\Delta x=x_{i+1 / 2}-x_{i-1 / 2}$ with the spatial index $i=0, \ldots, N_{x}$. The discrete time level $t^{n}$ are also uniformly spaced with a time step $\Delta t=t^{n+1}-t^{n}$, where $n$ is the temporal index. We denote the cell average in $\left[x_{i+1 / 2}, x_{x-1 / 2}\right]$ at time $t^{n}$ as

$$
\hat{\mathbf{u}}_{i}^{n}=\frac{1}{\Delta x} \int_{x_{i-1 / 2}}^{x_{i+1 / 2}} \hat{\mathbf{u}}\left(x, t^{n}\right) d x .
$$

- Relaxation step: In the relaxation step, we apply the central difference approximation in space and the backward Euler method in time:

$$
\left\{\begin{array}{l}
\hat{\mathbf{u}}_{i}^{*}=\hat{\mathbf{u}}_{i}^{n} \\
\frac{\hat{\mathbf{v}}_{i}^{*}-\hat{\mathbf{v}}_{i}^{n}}{\Delta t}=-\frac{1}{\epsilon}\left[\hat{\mathbf{v}}_{i}^{*}+\left(\mathbf{A}_{i}-\epsilon \alpha \mathbf{I}_{M}\right) \frac{\hat{\mathbf{u}}_{i+1}^{n}-\hat{\mathbf{u}}_{i-1}^{n}}{2 \Delta x}\right] .
\end{array}\right.
$$

- Convection step: In the convection step, we use the first order upwind method:

$$
\left\{\begin{array}{l}
\hat{\mathbf{u}}_{i}^{n+1}=\hat{\mathbf{u}}_{i}^{*}-\frac{\Delta t}{2 \Delta x}\left(\hat{\mathbf{v}}_{i+1}^{*}-\hat{\mathbf{v}}_{i-1}^{*}\right)+\frac{\sqrt{\alpha} \Delta t}{2 \Delta x}\left(\hat{\mathbf{u}}_{i+1}^{*}-2 \hat{\mathbf{u}}_{i}^{*}+\hat{\mathbf{u}}_{i-1}^{*}\right), \\
\hat{\mathbf{v}}_{i}^{n+1}=\hat{\mathbf{v}}_{i}^{*}-\alpha \frac{\Delta t}{2 \Delta x}\left(\hat{\mathbf{u}}_{i+1}^{*}-\hat{\mathbf{u}}_{i-1}^{*}\right)+\frac{\sqrt{\alpha} \Delta t}{2 \Delta x}\left(\hat{\mathbf{v}}_{i+1}^{*}-2 \hat{\mathbf{v}}_{i}^{*}+\hat{\mathbf{v}}_{i-1}^{*}\right) .
\end{array}\right.
$$

As $\epsilon \rightarrow 0,(4.6)$ gives

$$
\hat{\mathbf{v}}_{i}^{*}=-\mathbf{A}_{i} \frac{\hat{\mathbf{u}}_{i+1}^{n}-\hat{\mathbf{u}}_{i-1}^{n}}{2 \Delta x},
$$


which, when substituted into the first equation in the convection step (4.7), gives

$$
\hat{\mathbf{u}}_{i}^{n+1}=\hat{\mathbf{u}}_{i}^{n}-\Delta t \mathbf{A}_{i} \frac{\hat{\mathbf{u}}_{i+2}^{n}-2 \hat{\mathbf{u}}_{i}^{n}+\hat{\mathbf{u}}_{i-2}^{n}}{(2 \Delta x)^{2}}+\frac{\sqrt{\alpha} \Delta t}{2 \Delta x}\left(\hat{\mathbf{u}}_{i+1}^{n}-2 \hat{\mathbf{u}}_{i}^{n}+\hat{\mathbf{u}}_{i-1}^{n}\right) .
$$

This is the first order approximation, in both space and time, of the gPC diffusion equation (2.9). Thus, the fully discretized scheme is sAP.

REMARK 4.1. Here we adopted rather low-order discretizations in space and time to illustrate the procedure and the AP property of the fully discrete system. In practice, one can of course employ higher-order schemes, e.g., high-order TVD in space and high order implicit-explicit time discretization [28, 2, 18], and establish the corresponding sAP property.

5. Stochastic AP scheme for the random Carleman Model. We now consider the nonlinear random Carleman model (3.3). Except for the nonlinear term of the right-hand-side, the gPC Galerkin formulation for this system is similar to that of the random Goldstein-Taylor model. Again, let $\hat{\mathbf{u}}$ and $\hat{\mathbf{v}}$ be the coefficient vectors of the gPC expansion of $u$ and $v$, respectively. The gPC Galerkin procedure results in the following system

$$
\left\{\begin{array}{l}
\partial_{t} \hat{\mathbf{u}}+\partial_{x} \hat{\mathbf{v}}=0 \\
\partial_{t} \hat{\mathbf{v}}+\frac{1}{\epsilon} \partial_{x} \hat{\mathbf{u}}=-\frac{1}{\epsilon} \mathbf{B}(x, t) \hat{\mathbf{v}}
\end{array}\right.
$$

where the matrix $\mathbf{B}(x, t)=\left(b_{i j}(x, t)\right)_{1 \leq i, j \leq M}$ has entries

$$
\begin{aligned}
b_{i j}(x, t) & =\int \kappa(x, z) u_{N}(x, t, z) \Phi_{i}(z) \Phi_{j}(z) \rho(z) d z \\
& =\sum_{\ell=1}^{M} \hat{u}_{\ell} \int \kappa(x, z) \Phi_{\ell}(z) \Phi_{i}(z) \Phi_{j}(z) \rho(z) d z .
\end{aligned}
$$

5.1. The AP property. Let us first consider the limiting state (3.4) for the random Carleman model. Upon applying the ansatz (4.1) in the first equation of (3.4) and conducting the Galerkin projection, we obtain

$$
\hat{\mathbf{v}}=-\mathbf{B}^{-}(x, t) \partial_{x} \hat{\mathbf{u}}
$$

where the matrix $\mathbf{B}^{-}=\left(b_{i j}^{-}\right)_{1 \leq i . j \leq M}$ has entries

$$
b_{i j}^{-}(x, t)=\int \frac{1}{\kappa(x, z) u_{N}(x, t, z)} \Phi_{i}(z) \Phi_{j}(z) \rho(z) d z .
$$

If one uses this in (3.4) to derive the gPC limiting equation, then the scheme gives a complicated nonlinear algebraic system. Alternatively, we write the first equation of (3.4) in an equivalent form

$$
\kappa(x, z) u v=-\partial_{x} u
$$

which renders its gPC Galerkin projection

$$
\mathbf{B} \hat{\mathbf{v}}=-\partial_{x} \hat{\mathbf{u}},
$$


where $\mathbf{B}$ is defined in (5.2). This yields

$$
\hat{\mathbf{v}}=-\mathbf{B}^{-1} \partial_{x} \hat{\mathbf{u}} .
$$

Thus the gPC equation for limiting problem (3.4) is

$$
\partial_{t} \hat{\mathbf{u}}=\partial_{x}\left[\mathbf{B}^{-1}(x, t) \partial_{x} \hat{\mathbf{u}}\right] .
$$

We now consider the diffusive limit of the formulation (5.1). As $\epsilon \rightarrow 0^{+}$, the second equation leads to

$$
\hat{\mathbf{v}}=-\mathbf{B}^{-1}(x, t) \partial_{x} \hat{\mathbf{u}} .
$$

Substituting into the first equation of (5.1), we obtain exactly (5.7). Therefore, (5.1) is sAP.

5.2. Connection with the relaxation scheme for nonlinear diffusion equation (3.4). As discussed in the previous section, the gPC approximation to the nonlinear diffusion equation (3.4) is difficult. Yet, the above discussion of the AP property has an analogy to the Jin-Xin relaxation scheme for nonlinear hyperbolic conservation laws ([21]), in the sense that the nonlinear diffusion equation (3.4) is approximated by the semi-linear hyperbolic equations (the Carleman model) (3.3), which can be more easily solved numerically than the original equation (3.4). To be precise, we start with the gPC Galerkin system (5.1), which, upon taking the relaxation limit $\epsilon \rightarrow 0$, leads to an effective numerical scheme for (3.4).

The scheme takes the following form,

Convection step:

$$
\frac{\hat{\mathbf{u}}^{n+1}-\hat{\mathbf{u}}^{n}}{\Delta t}+\frac{\hat{\mathbf{v}}_{j+1 / 2}^{n}-\hat{\mathbf{v}}_{j-1 / 2}^{n}}{\Delta x}=0,
$$

Relaxation step:

$$
\hat{\mathbf{v}}_{j+1 / 2}^{n}=-\left(\mathbf{B}_{j+1 / 2}^{n}\right)^{-1} \frac{\hat{\mathbf{u}}_{j+1}^{n}-\hat{\mathbf{u}}_{j}^{n}}{\Delta x},
$$

where $\mathbf{B}_{j+1 / 2}=\frac{1}{2}\left[\mathbf{B}_{j}+\mathbf{B}_{j+1}\right]$.

By substituting (5.9) into (5.8), we obtain

$$
\frac{\hat{\mathbf{u}}^{n+1}-\hat{\mathbf{u}}^{n}}{\Delta t}=\frac{1}{(\Delta x)^{2}}\left[\left(\mathbf{B}_{j+1 / 2}^{n}\right)^{-1}\left(\hat{\mathbf{u}}_{j+1}^{n}-\hat{\mathbf{u}}_{j}^{n}\right)-\left(\mathbf{B}_{j-1 / 2}^{n}\right)^{-1}\left(\hat{\mathbf{u}}_{j}^{n}-\hat{\mathbf{u}}_{j-1}^{n}\right)\right] .
$$

Clearly, this is a second order space approximation for (3.4).

6. Stochastic AP scheme for the random linear transport equation. We now discuss the gPC Galerkin formulation for the linear transport equation (3.8) with random cross-section $\sigma(x, z)$. Let

$$
r_{N}(x, z, t)=\sum_{m=1}^{M} \hat{r}_{m}(t, x) \Phi_{m}(z), \quad j_{N}(x, z, t)=\sum_{m=0}^{M} \hat{j}_{m}(t, x) \Phi_{m}(z)
$$

be the $N$ th-order gPC expansion for the solutions and

$$
\hat{\mathbf{r}}=\left(\hat{r}_{1}, \cdots, \hat{r}_{M}\right)^{T}, \quad \hat{\mathbf{j}}=\left(\hat{j}_{1}, \cdots, \hat{j}_{M}\right)^{T},
$$


be the expansion coefficient vectors. The gPC Galerkin procedure can be applied to (3.8) in a straightforward manner and results in the following system:

$$
\left\{\begin{array}{l}
\partial_{t} \hat{\mathbf{r}}+v \partial_{x} \hat{\mathbf{j}}=\frac{1}{\epsilon^{2}} \mathbf{S}(x)(\overline{\mathbf{r}}-\hat{\mathbf{r}}), \\
\partial_{t} \hat{\mathbf{j}}+\frac{v}{\epsilon^{2}} \partial_{x} \hat{\mathbf{r}}=-\frac{1}{\epsilon^{2}} \mathbf{S}(x) \hat{\mathbf{j}}
\end{array}\right.
$$

where

$$
\overline{\mathbf{r}}(x, t)=\int_{0}^{1} \hat{\mathbf{r}} d v
$$

and $\mathbf{S}(x)=\left(s_{i j}(x)\right)_{1 \leq i, j \leq M}$ is a $M \times M$ matrix with entries

$$
s_{i j}(x)=\int \sigma(x, z) \Phi_{i}(z) \Phi_{j}(z) \rho(z) d z .
$$

The remaining AP discretizations for time and space are similar to that of the Goldstein-Taylor model and the Carleman model. For velocity discretization, we use the discrete-ordinate method, i.e., we choose the discrete velocity $v_{k}$ to be the Legendre-Gauss quadrature points and integrate $\overline{\mathbf{r}}$ on the right hand side of (6.2) using Gauss's quadrature rule. See, for example, [17]. The fully discretized scheme can be shown to be AP, similar to the two previous models. We omit the details.

7. Stochastic collocation methods. We now discuss stochastic collocation methods using the general setup from Section 2. In stochastic collocation, one first chooses a set of collocation nodes/samples for the input random variable $z \in \mathbb{R}^{d}$, $d \geq 1$. Let $\left\{z^{(j)}\right\}_{j=1}^{N_{s}} \subset I_{z}$ be the set of nodes, where $N_{s}$ is the total number of nodes, one then applies a deterministic AP scheme to (2.3) for each fixed $z^{(j)}$, for $j=1, \ldots, N_{s}$, and obtain the solution ensemble $v_{j}^{\epsilon}(x, t)=v^{\epsilon}\left(x, t, z^{(j)}\right)$. This is a straightforward non-intrusive procedure and poses no coding difficulty.

Equipped with the solution ensembles $\left\{z^{(j)}, v_{j}^{\epsilon}(x, t)\right\}_{j=1}^{N_{s}}$, one then, in the postprocessing step, seeks to construct an accurate (gPC) approximation $v^{\epsilon}(x, t, z)$. Most of the construction procedures are linear and render an approximation such as,

$$
v^{\epsilon}(x, t, z)=\sum_{j=1}^{N_{s}} v_{j}^{\epsilon}(x, t) \ell_{j}(z),
$$

where the form of the function $\left\{\ell_{j}(z)\right\}$ depends on the construction method. For example, if the Lagrange interpolation method is used, then $\ell_{j}\left(z^{(i)}\right)=\delta_{i j}$. For other construction methods, e.g., least-square regression, discrete projection, etc., the functions are more involved but can be constructed nevertheless. (For an overview of stochastic collocation methods, see, for example, [32, 33].) Upon taking the diffusive limit $\epsilon \rightarrow 0$, we obtain

$$
v(x, t, z)=\sum_{j=1}^{N_{s}} v_{j}(x, t) \ell_{j}(z),
$$

where $v_{j}(x, t)=\lim _{\epsilon \rightarrow 0} v_{j}^{\epsilon}(x, t)$ is the limit of each sample solution and is deterministically AP. 
If (7.1) is constructed via an interpolation procedure, the limiting solution (7.2) satisfies $v\left(x, t, z^{(j)}\right)=v_{j}(x, t)$, which means the method satisfies the limit equation (2.4) at each collocation nodes $z^{(j)}$. And this leads to a strongly AP scheme if the nodes fills up the random space $I_{z}$ asymptotically. This is obvious not a practical situation. For locations outside the sample set, it can be argued that the method is AP subject to interpolation errors. Controlling interpolation errors is, however, a quite open research topic, even for smooth problems. And the interpolation errors can be highly non-trivial for high dimensional random space. Although it is possible to control the errors using tensor Gauss nodes or sparse grids ([34]), they lead to fast growth of the number of nodes in high dimensions and can be impractical to use.

If the construction (7.1) is not of interpolation type, e.g., regression, then it will not be AP at the sample nodes. On the other hand, except in some rare cases, it is also not possible to ensure (7.2) solves the limit problem (2.4) in a weak form. And therefore it will not sAP, weakly or strongly. This is in contrary to the gPC Galerkin methods presented here - the limiting gPC Galerkin solutions satisfy the limiting problem in a Galerkin weak form, for any given order of the polynomial expansions. Consequently, it is fair to state that the gPC Galerkin approach is much preferred than the collocation approach, if one wishes to enforce AP properties in the random space.

8. Numerical Examples. In this section, we present several numerical examples to illustrate the effectiveness of our method. We typically use reference solutions to examine the performance of the numerical solutions. To examine the accuracy, two metrics are used to quantify the errors: the differences in the mean solutions and in the corresponding standard deviation, with $L^{2}$ norm in $x$ :

$$
\begin{aligned}
& e_{\text {mean }}(t)=\left\|\mathbb{E}\left[u^{h}\right]-\mathbb{E}[u]\right\|_{L^{2}}, \\
& e_{\text {std }}(t)=\left\|\sigma\left[u^{h}\right]-\sigma[u]\right\|_{L^{2}},
\end{aligned}
$$

where $u^{h}, u$ are the numerical solutions and the reference solutions, respectively. The reference solutions for the mean and standard deviation are typically computed by high-order stochastic collocation methods, using either tensor Gauss quadrature or sparse grids quadrature.

8.1. The Goldstein-Taylor Model. We first consider the Goldstein-Taylor equation (3.1) with the following initial data

$$
\begin{array}{lll}
u(x, z, 0)=2.0, & v(x, z, 0)=0, & x \in[-1,0], \\
u(x, z, 0)=1.0, & v(x, z, 0)=0, & x \in(0,1],
\end{array}
$$

and random coefficient

$$
a(x, z)=2+z, \quad z \in[-1,1] .
$$

This makes the problem one dimension in random space, and allows us to examine carefully the error behavior. The spatial and temporal discretizations discussed in Section 4 are used.

We first set $\epsilon=0.49$ to be a relatively large number. The mean (left) and standard deviation (right) of the solution until $t=0.25$ at order $N=4$ are plotted in Fig. 8.1, where we also show the mean and standard deviation obtained by the stochastic collocation method over 20 Legendre-Gauss quadrature points. Good agreement can 

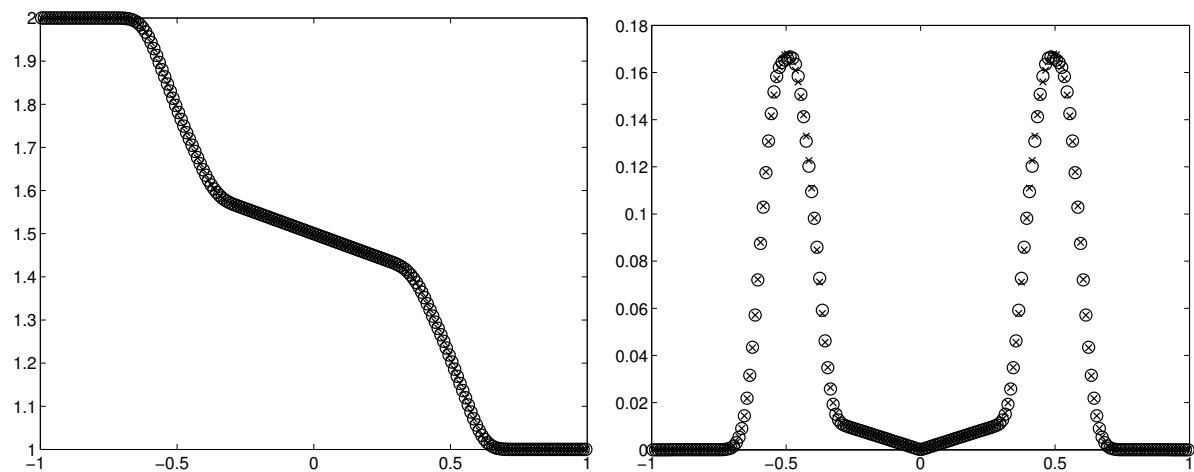

FIG. 8.1. The Goldstein-Taylor Model: The mean (left) and standard deviation (right) of $u$ with $\epsilon=0.49$, obtained by 4th-order gPC Galerkin (circles) and stochastic collocation (crosses).

be seen between the two sets of results. This gives us a qualitative understanding of the solution behavior.

We then consider the case of very small $\epsilon$ by setting $\epsilon=10^{-12}$. In this case, the analytical solution of the limiting random diffusion equation (3.2) is

$$
u(x, t, z)=\frac{3}{2}-\frac{1}{2} \operatorname{erf}\left(\frac{x}{\sqrt{4 a(z) t}}\right) .
$$

This is an accurate approximation to the exact solution, with error $\sim O(\epsilon)$. The solution profiles of the mean and standard deviations are shown in Fig. 8.2, where we again visually observe good agreement among the 4th-order gPC solutions, stochastic collocations and the analytical solution (8.2). In Fig. 8.3, the errors at time $t=0.02$ with respect to increasing $\mathrm{gPC}$ order are plotted, at various levels of grid resolutions, $\Delta x=0.04$ (squares), $\Delta x=0.02$ (circles), and $\Delta x=0.01$ (stars). The time step is $\Delta t=\Delta x^{2} / 12$. The fast exponential convergence with respect to the order of gPC expansion can be observed. The errors quickly saturate at modest gPC orders and the saturation levels become smaller with finer $\Delta x$. This indicates that in this case the errors have a larger contribution from the temporal and spatial discretization than that from the gPC expansion. The advantage of AP methods can be seen here: even for such an exceedingly small parameter $\epsilon=10^{-12}$, accurate solutions can still be obtained with very modest mesh.

While keeping the discretization in $x$ at the same three different levels, we now vary the value of $\epsilon$ and examine the solutions. We keep the stochastic AP scheme at $N=4 \mathrm{gPC}$ order. From the results above, it is obvious that at $N=4$ the errors in random space is subdominant compared to those in space and time. Hence, the numerical solutions can be considered accurate up to the discretization errors, whereas (8.2) is accurate up to $O(\epsilon)$ error. In Fig.8.4, we show the differences of the mean (solid lines) and standard deviation (dash lines) between the analytical solution (8.2) and the 4th-order gPC solutions with respect to varying values of $\epsilon$. We observe the reduction of the differences as $\epsilon$ becomes smaller, as the analytical solution (8.2) becomes more accurate, before they become saturated as the numerical errors in the sAP solutions start to dominate.

To further demonstrate the effectiveness of the sAP method, we now consider a case of spatially varying $\epsilon(x)>0$ spanning a wide range of mixing scales. Specifically, 

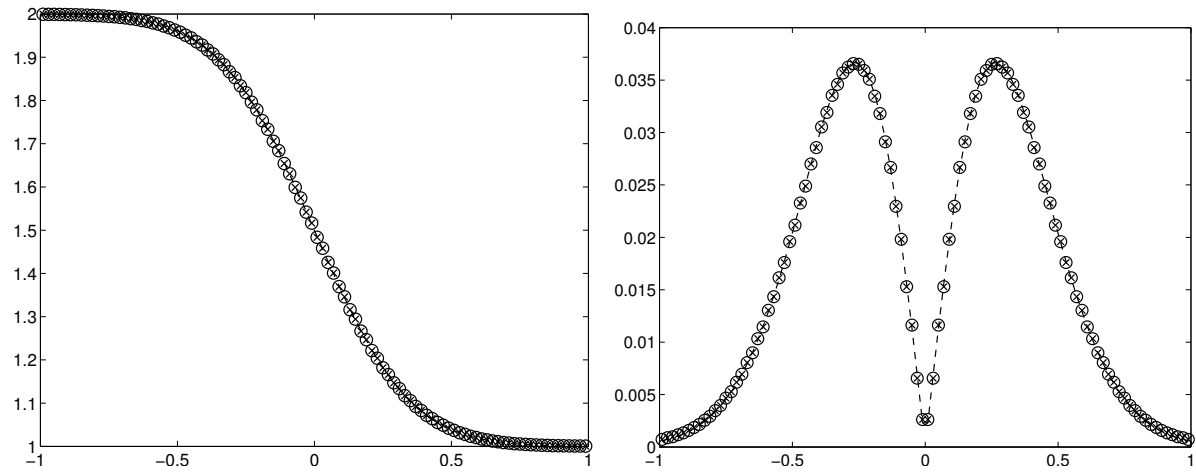

FIG. 8.2. The Goldstein-Taylor Model: The mean (left) and standard deviation (right) of $u$ with $\epsilon=10^{-12}$, obtained by 4th-order gPC Galerkin (circles) and stochastic collocation (crosses), along with the analytical solution (8.2) (dashed lines) of the limiting diffusion equation (3.2).

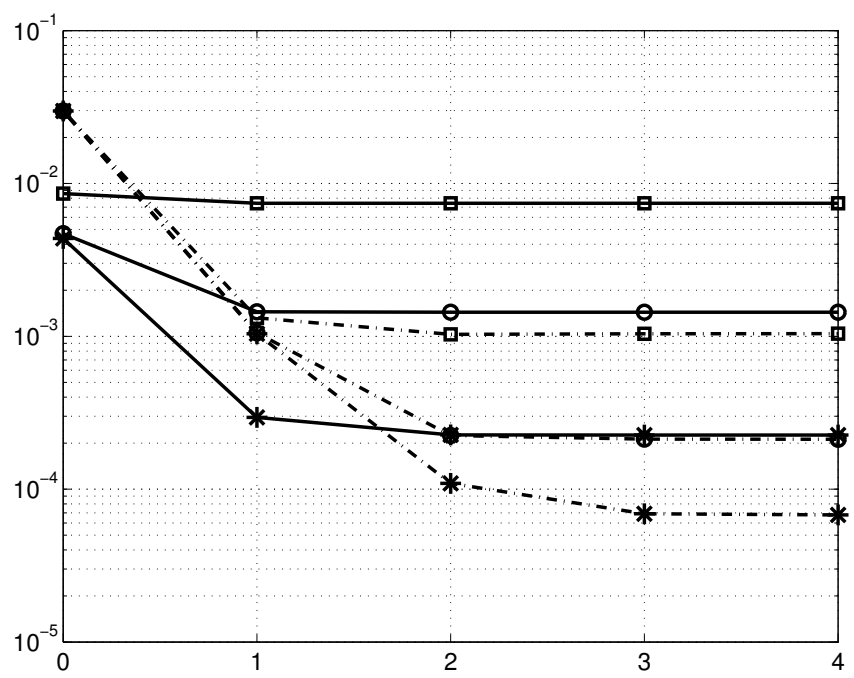

FIG. 8.3. The Goldstein-Taylor Model: Error convergence in mean (solid lines) and standard deviation (dashed lines), with respect to gPC order for $\epsilon=10^{-12}$. (Squares: $\Delta x=0.04$, circles: $\Delta x=0.02$, stars: $\Delta x=0.01$.

we consider the following

$$
\epsilon(x)=\epsilon_{0}+\frac{1}{2}\left[\tanh \left(1-\frac{11}{2} x\right)+\tanh \left(1+\frac{11}{2} x\right)\right]
$$

which is similar to the one used in [7] for simulations of deterministic Boltzmann equation. As seen from Fig.8.5, the $\epsilon(x)$ varies smoothly from $\epsilon_{0}$ to $\sim O(1)$. Here we set $\epsilon_{0}=10^{-12}$,

The multiscale nature of the problem requires good accuracy over the entire range of $\epsilon$, which our sAP method is able to deliver. The mean and standard deviation of the solution $u$ at $t=0.02$ with $\Delta x=0.01$ and $\Delta t=\frac{1}{12} \Delta x^{2}$ are shown in Fig. 8.6. We observed good agreement between the sAP gPC-Galerkin solution at order $N=4$ and the reference solution. The reference solution is computed by the stochastic collocation method using 20 one-dimensional Legendre-Gauss quadrature points and 


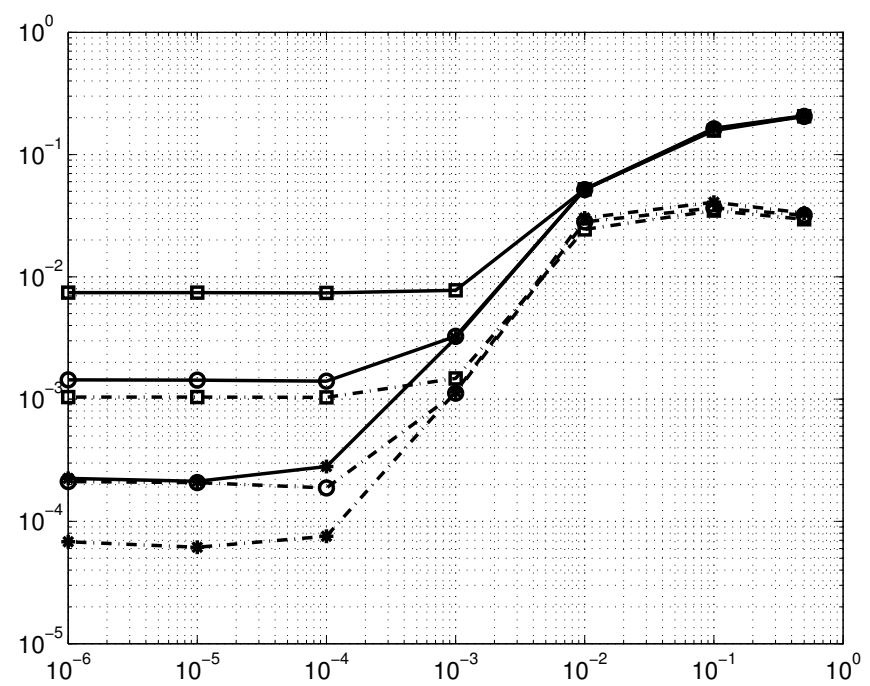

FIG. 8.4. The Goldstein-Taylor Model: Differences in mean (solid lines) and standard deviation (dashed lines) with respect to $\epsilon$ between the analytical solution (8.2) of the limiting diffusion equation (3.2) and the 4th-order $g P C$ solution with $\Delta x=0.04$ (squares), $\Delta x=0.02$ (circles), and $\Delta x=0.01$ (stars).

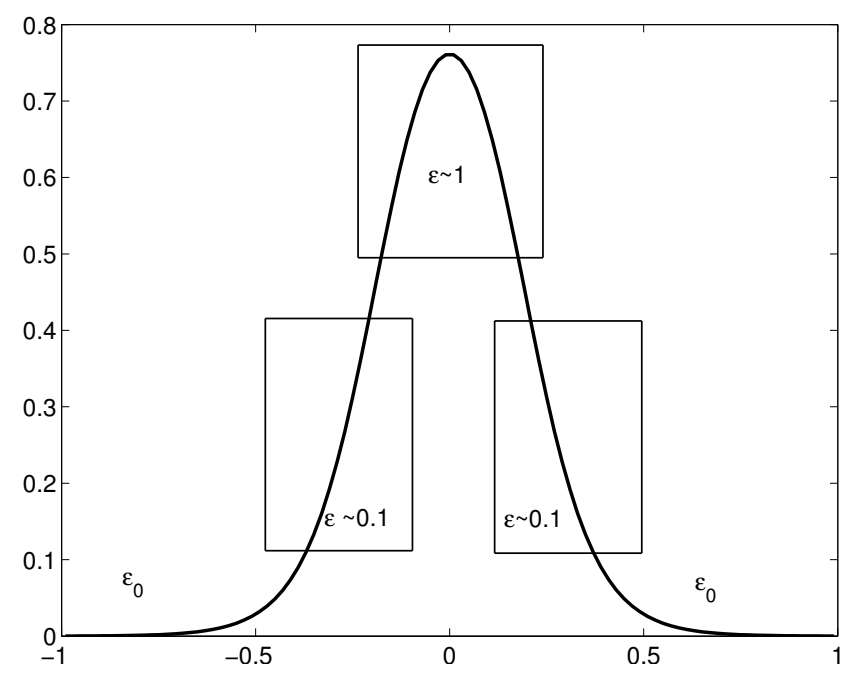

FIG. 8.5. The Goldstein-Taylor Model: illustration of the spatially varying $\epsilon(x)$ over the physical domain.

also based on the first order deterministic AP method in Section 4 with the same $\Delta x$ and $\Delta t$. The errors in the gPC-Galerkin solutions are plotted in Fig. 8.7, where the exponential convergence is clearly observed with increasing order of gPC expansion.

8.2. The Carleman Model. We now consider the nonlinear Carleman model and the corresponding stochastic AP scheme (see section 5). We focus on the diffusive regime and fix $\epsilon=10^{-12}$.

In the first case study, we employ the same random coefficient, initial data and boundary condition as in the previous section. Lacking an analytical solution, we em- 

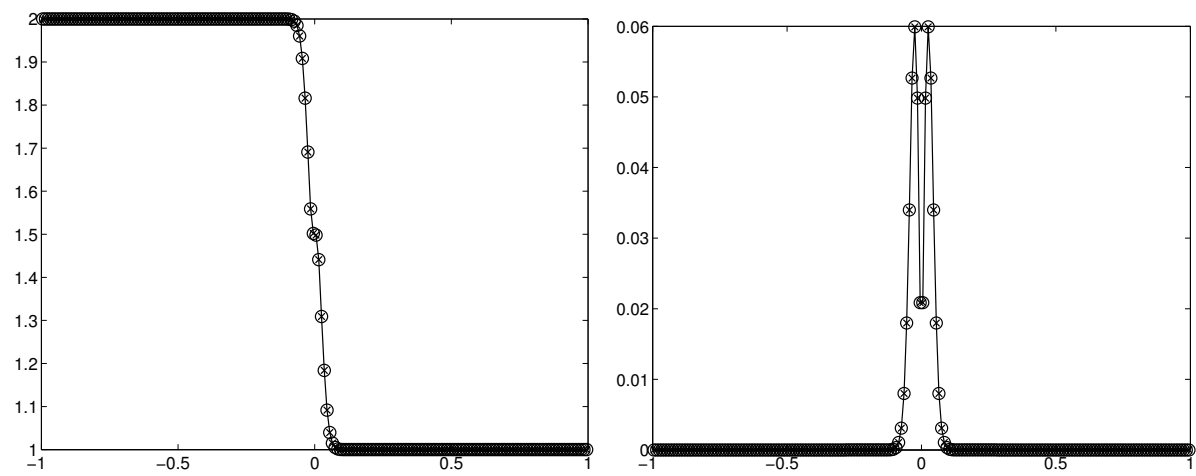

FIG. 8.6. The Goldstein-Taylor Model with the spatially varying $\epsilon(x)$ : The mean (left) and standard deviation (right) of $u$ with $\epsilon_{0}=10^{-12}$, obtained by 4th-order gPC Galerkin (circles) and stochastic collocation (crosses).

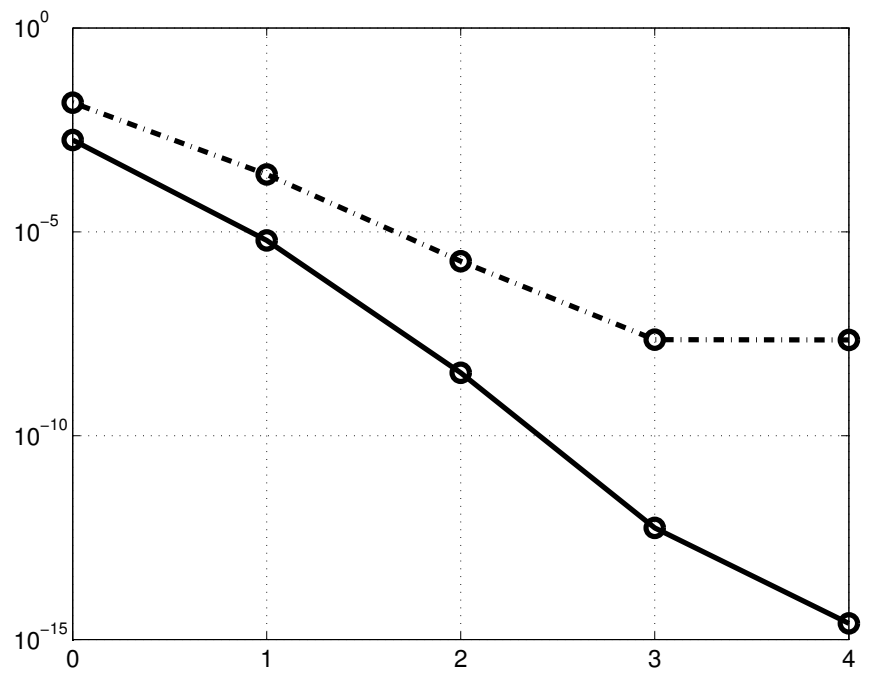

FIG. 8.7. The Goldstein-Taylor Model with the spatially varying $\epsilon(x)$ : Error convergence in mean (solid lines) and standard deviation (dashed lines), with respect to gPC order for $\epsilon_{0}=$ $10^{-12}, \Delta x=0.01, \Delta t=\frac{1}{12} \Delta x^{2}$.

ploy the high-order stochastic collocation method with 20 Legendre-Gauss quadrature points to compute the reference mean and standard deviation solutions. In Fig. 8.8, the errors of the mean (solid lines) and standard deviation (dash lines) of $u$ (circle) and $v$ (triangle) are plotted at increasing gPC expansion order. Spectral convergence is clearly observed. All of the numerical solutions are computed until $t=0.04$ with $\Delta x=0.02$ and $\Delta t=\frac{1}{12} \Delta x^{2}$.

In Fig. 8.9, we plot the mean and standard deviation of $u$, obtained by the 4thorder gPC Galerkin and collocation, as well as a "semi-exact" solution. Here the "semi-exact" solution is obtained by solving the limiting nonlinear diffusion equation (3.4) by the stochastic collocation method over 20 one dimensional Legendre-Gauss quadrature points, using the Euler forward in time and central difference in space with the same $\Delta t$ and $\Delta x$. Good agreement can be observed among these different 
methods.

We now examine the difference between our gPC-AP solution at $t=0.04$ with $\Delta x=0.02, \Delta t=\frac{1}{12} \Delta x^{2}$ and the numerical solution from the limiting nonlinear diffusion equation (3.4). This is shown in Fig. 8.10, at different values of $\epsilon$. The errors clearly decrease as $\epsilon$ becomes smaller, as expected, before they saturate at a level where the numerical errors become dominant.

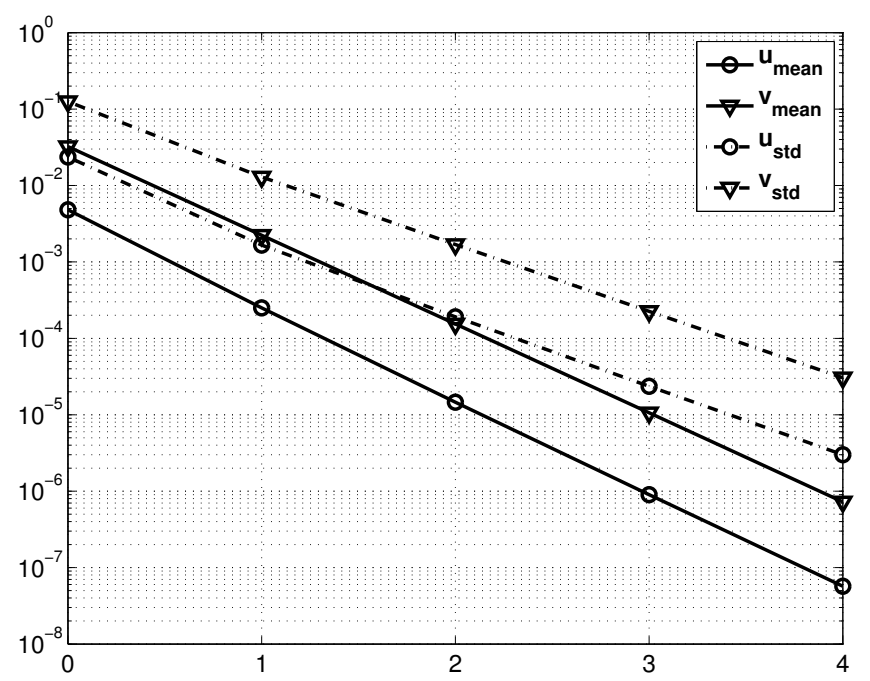

FIG. 8.8. The Carleman Model: Error convergence with respect to $g P C$ order for $\epsilon=10^{-12}$.
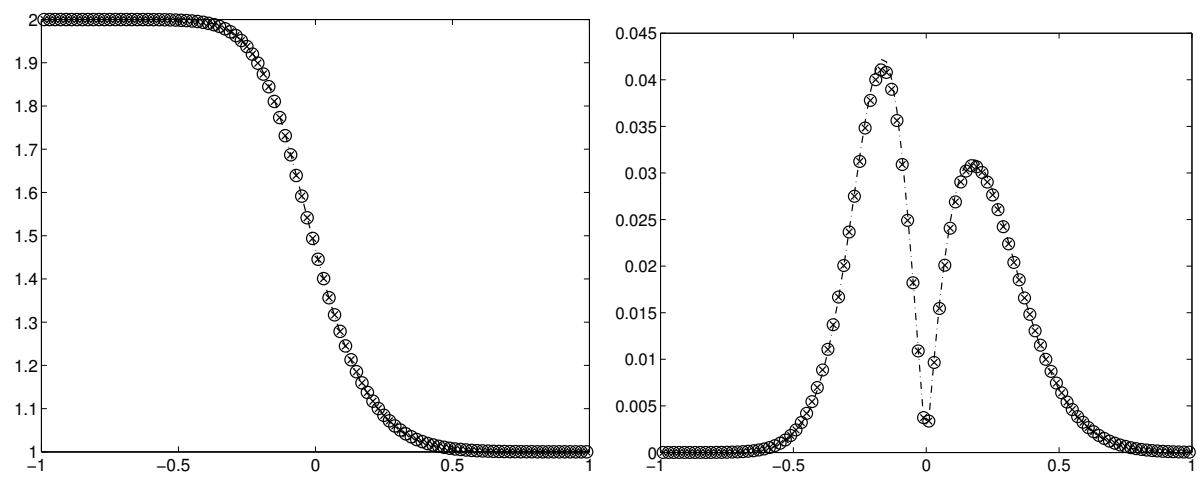

FIG. 8.9. The Carleman Model: The mean (left) and standard deviation (right) of $u$ at $\epsilon=$ $10^{-12}$, obtained by $N=4$ order gPC Galerkin (circles), the stochastic collocation method (crosses), and the limiting random diffusion equation (3.4) (dashed line).

We now consider a more general case, where the random input $\kappa$ is a random field with the following form,

$$
\kappa(x, z)=1+\sigma \sum_{i=1}^{d} \frac{1}{i \pi} \cos (2 \pi i x) z_{i} .
$$

This resembles the form of the well known Karhunen-Loeve expansion, widely used for modeling random fields. For benchmarking purpose, we set $\sigma=2, d=2$. 


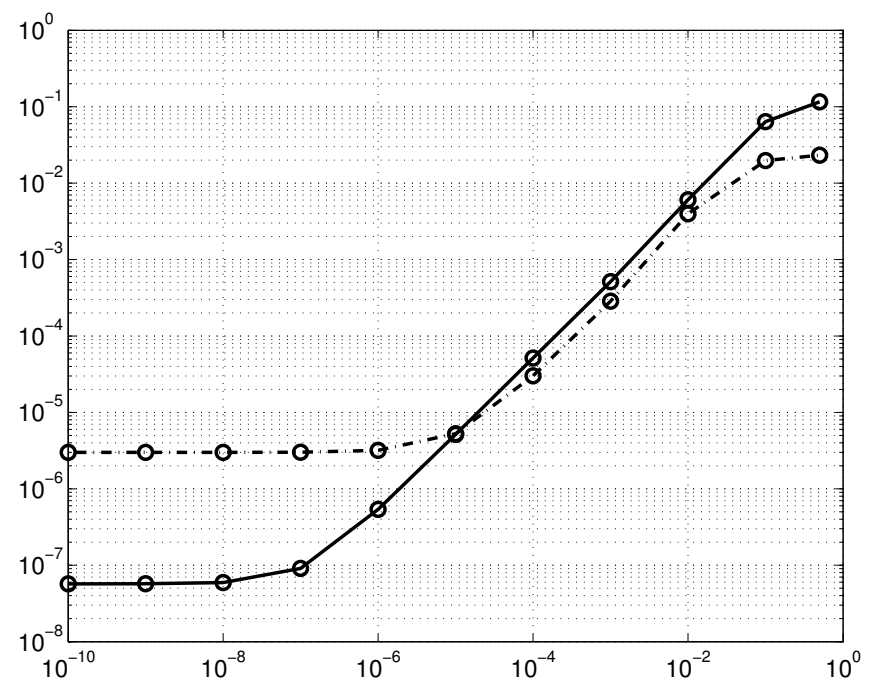

FIG. 8.10. The Carleman Model: Errors in the mean (solid line) and standard deviation (dash line) of $u$ by the gPC solution with order $N=4$ at varying $\epsilon$ values. The reference solution is obtained by solving the limiting random diffusion equation (3.4).

The mean and standard deviation of the solution are shown in Fig. 8.11, where we observe good agreement between the AP gPC-Galerkin at order $N=5$ and the reference solutions obtained a stochastic collocation over $8^{d}$ tensor Legendre-Gauss quadrature points. The errors in the gPC-Galerkin solutions are plotted in Fig.8.12, where again we observe the expected exponential convergence. All of the numerical solutions are computed until $t=0.02$ with a modest mesh of $\Delta x=0.02$ and $\Delta t=$ $\frac{1}{12} \Delta x^{2}$.
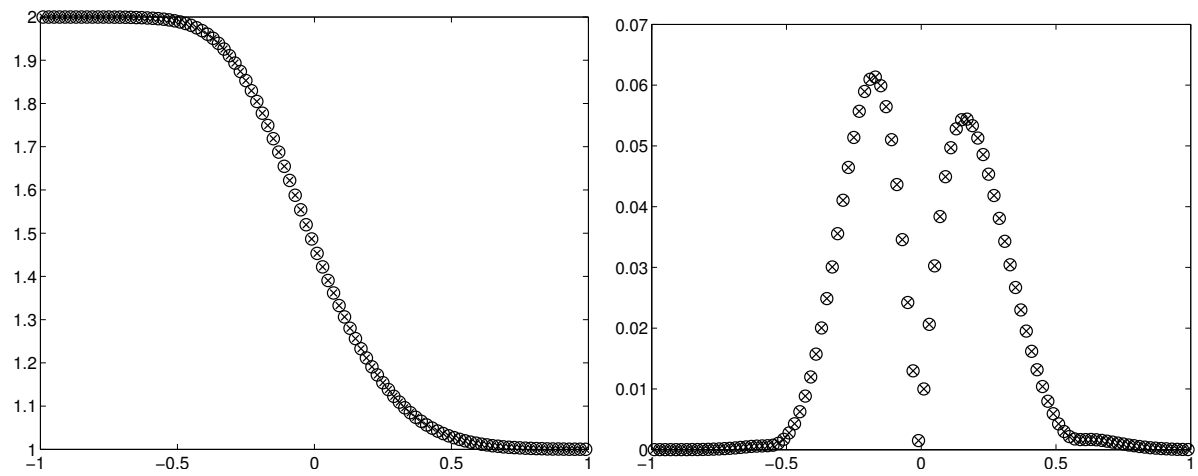

FIG. 8.11. The Carleman Model: Mean (left) and standard deviation (right) of $u$ by $g P C$ Galerkin with order $N=5$ (circles) and the stochastic collocation method (crosses) for $\epsilon=10^{-12}$.

8.3. The linear transport equation. We now consider the linear transport equation (3.8) with the random coefficient $\sigma=2+z$, where $z \in[-1,1]$ is uniformly distributed. The initial conditions are

$$
r(x, v, z, 0)=0, \quad j(x, v, z, 0)=0,
$$




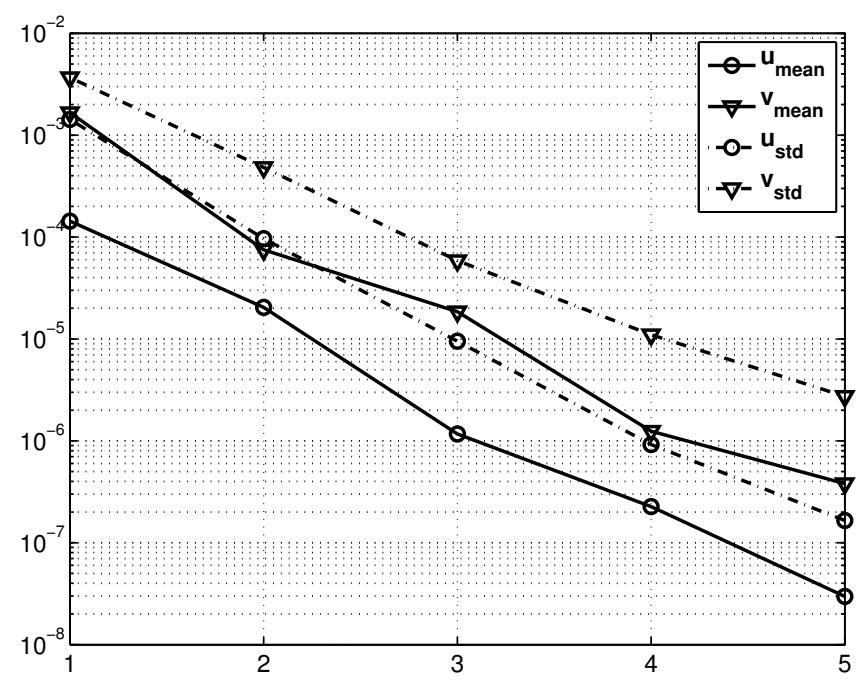

FIG. 8.12. The Carleman Model: Errors with respect to gPC Galerkin order for $\epsilon=10^{-12}$.

and the boundary conditions are (see [17])

$$
\sigma j=-v r_{x} .
$$

and

$$
r-\left.\frac{\epsilon}{\sigma} v r_{x}\right|_{x=0}=1, \quad r+\left.\frac{\epsilon}{\sigma} v r_{x}\right|_{x=1}=0 .
$$

The analytical solution for the limiting random diffusion equation (3.9) with the above initial and boundary condition in the diffusive regime is

$$
\bar{r}(x, t, z)=1-\operatorname{erf}\left(\frac{x}{\sqrt{\frac{4}{3 \sigma} t}}\right) .
$$

When $\epsilon$ is small, we use this as the reference solution, as it is accurate with an error of $O\left(\epsilon^{2}\right)$. Hereafter we set $\epsilon=10^{-8}$. In addition, the standard 16-points Gauss-Legendre quadrature set is used for the velocity space to compute $\bar{r}$ in (6.2) in the following example.

In Fig. 8.13, we plot the errors in mean and standard deviation of the gPC numerical solutions at $t=0.01$ with different gPC orders. Three sets of results are included: solutions with $\Delta x=0.04$ (squares), $\Delta x=0.02$ (circles), $\Delta x=0.01$ (stars) and $\Delta t=0.0002 / 3$. We observe that the errors become smaller with finer mesh. And at the finest mesh with $\Delta x=0.01$, fast convergence with respect to the $\mathrm{gPC}$ expansion order can be seen. It is then obvious that the errors due to gPC expansion can be neglected at order $N=4$. The solution profiles of the mean and standard deviation are shown on the left and right of Fig. 8.14, respectively. Here we observe good agreement among the gPC-Galerkin sAP method, stochastic collocation method with 20 Gauss-Legendre quadrature points, and the analytical solution (8.6).

In Fig. 8.15, we examine the difference between the solution $t=0.01$ obtained by the 4th-order gPC AP method with $\Delta x=0.01, \Delta t=\frac{1}{12} \Delta x^{2}$ and the limiting 


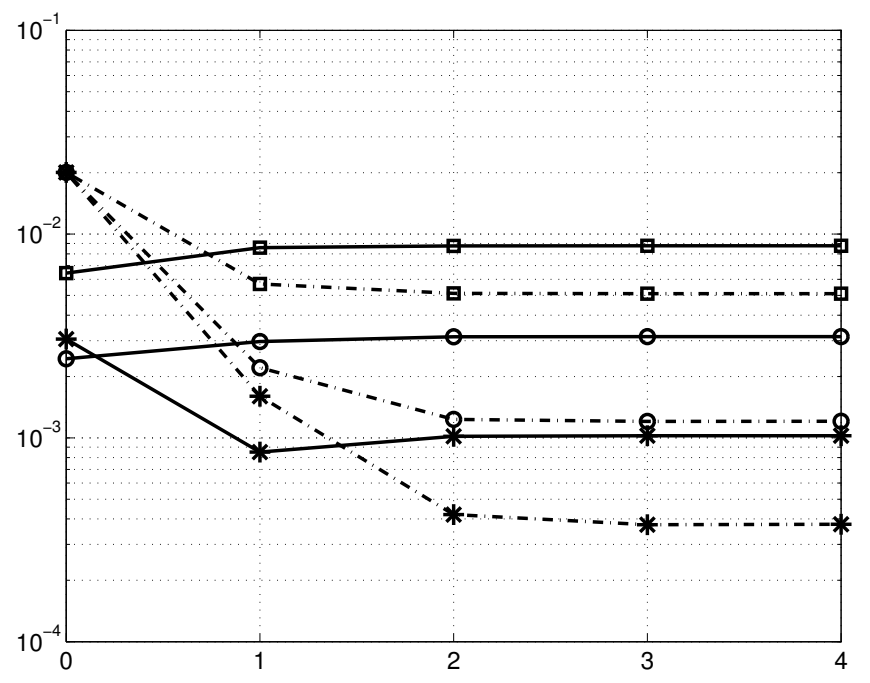

FIG. 8.13. The linear transport equation: Errors of the mean (solid line) and standard deviation (dash line) of $\bar{r}$ (circle) with respect to the gPC order at $\epsilon=10^{-8}: \Delta x=0.04$ (squares), $\Delta x=0.02$ (circles), $\Delta=0.01$ (stars).
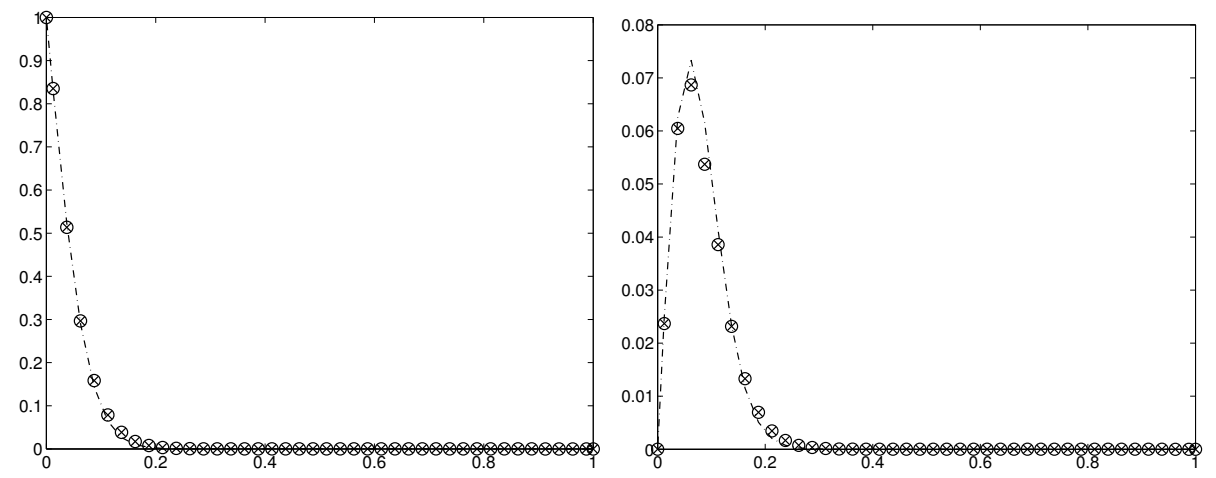

FIG. 8.14. The linear transport equation: The mean (left) and standard deviation (right) of $\bar{r}$ at $\epsilon=10^{-8}$, obtained by the gPC Galerkin at order $N=4$ (circles), the stochastic collocation method (crosses), and the limiting analytical solution (8.6).

analytical solution (8.6). As expected, we observe the differences become smaller as $\epsilon$ is smaller, before they saturate at a level where the numerical errors in the gPC sAP solutions become dominant.

We then model the random input as a random field, similar to the previous example, in the following form,

$$
\sigma(x, z)=1+\sigma \sum_{i=1}^{d} \frac{1}{(i \pi)^{2}} \cos (2 \pi i x) z_{i},
$$

where we set $\sigma=4$, and $d=5$. This represents a modestly high dimensional random inputs. The mean and standard deviation of the solution $\bar{r}$ at $t=0.01$ obtained by the 5th-order gPC Galerkin with $\Delta x=0.025, \Delta t=0.0002 / 3$ are shown in Fig. 8.16. We then use the high-order stochastic collocation method over 5 -dimensional sparse 


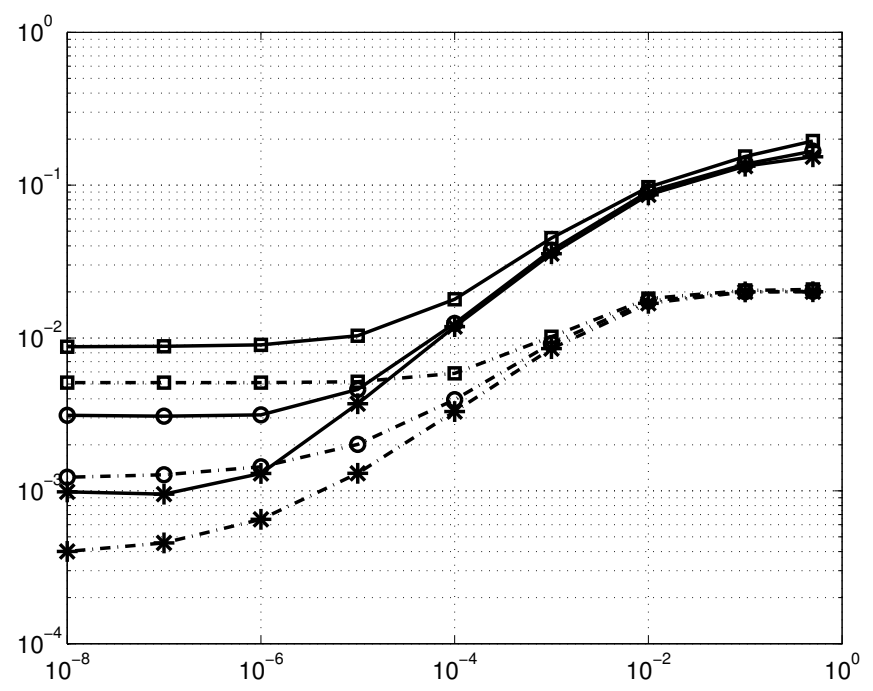

FIG. 8.15. The linear transport equation: Differences in the mean (solid line) and standard deviation (dash line) of $\bar{r}$ with respect to $\epsilon^{2}$, between the limiting analytical solution (8.6) and the 4th-order gPC solution with $\Delta x=0.04$ (squares), $\Delta x=0.02$ (circles) and $\Delta x=0.01$ (stars).

quadrature points with level 5 Clenshaw-Curtis rules (cf., [34]) to compute the reference mean and standard deviation of the solutions. In Fig. 8.17, we show the errors of the mean (solid lines) and standard deviation (dash lines) of $\bar{r}$ with respect to the order of gPC expansion. The fast exponential convergence of the errors can be clearly seen.

To illustrate the effectiveness of the sAP method, we also computed the mean and standard deviation of the solution $\bar{r}$ for $d=10$. This represents a relatively high dimensional stochastic problem. The results are shown in Fig.8.18, where we observed good agreement between the sAP gPC-Galerkin solution at the order $N=3$ and the reference solution obtained by the level 2 Clenshaw-Curtis sparse grids solution. Detailed error convergence requires much higher resolutions and is not practical for such a high dimensional problem $(d=10)$.
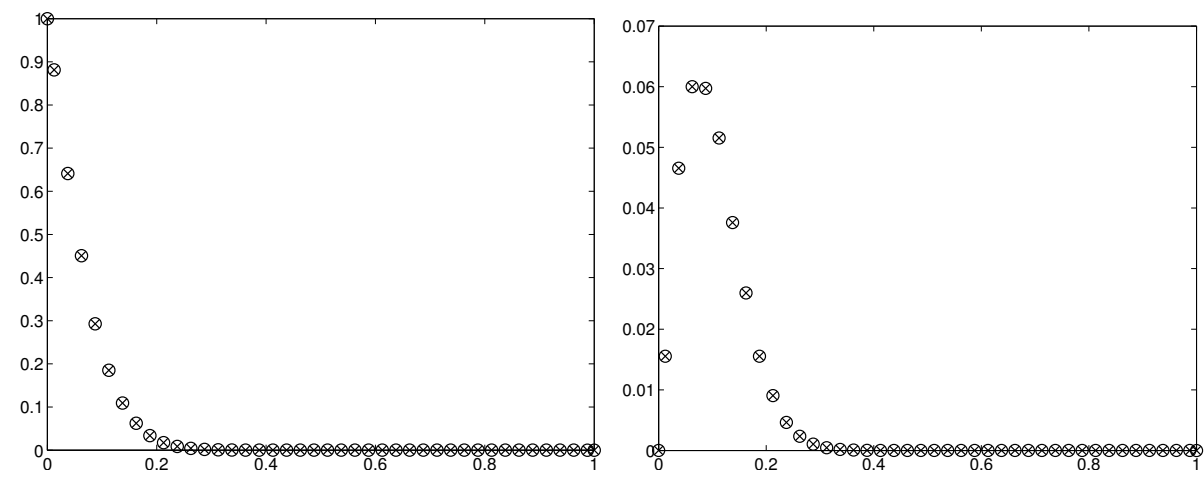

FIG. 8.16. The linear transport equation: The mean (left) and standard deviation (right) of $\bar{r}$ at $\epsilon=10^{-8}$, obtained by 5th-order gPC Galerkin (circles) and the stochastic collocation method (crosses). The randome input has dimension $d=5$. 


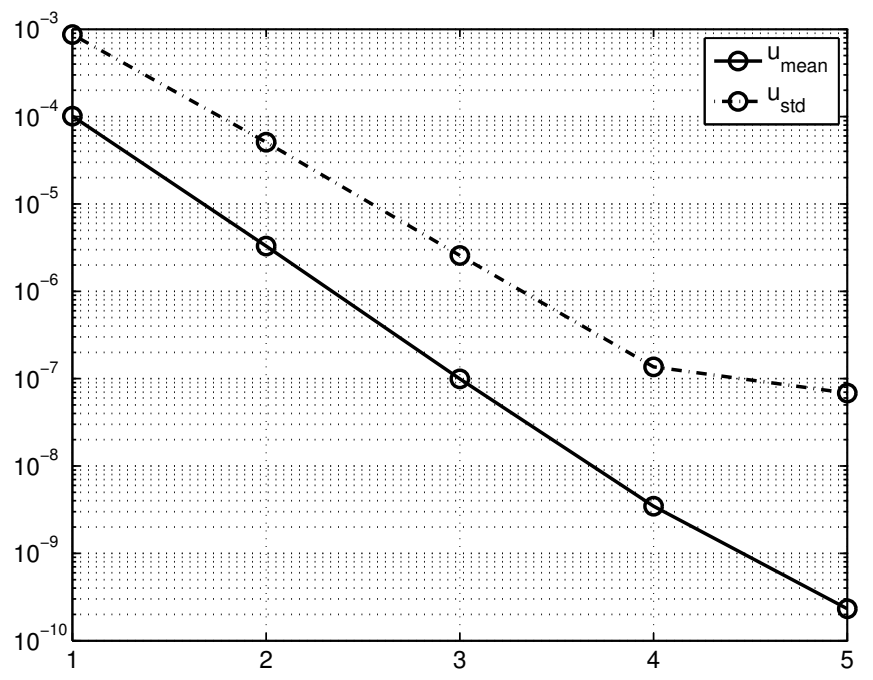

FIG. 8.17. The linear transport equation: Errors of the mean (solid line) and standard deviation (dash line) of $\bar{r}$ with respect to $g P C$ order, with the $d=5$ dimensional random input.
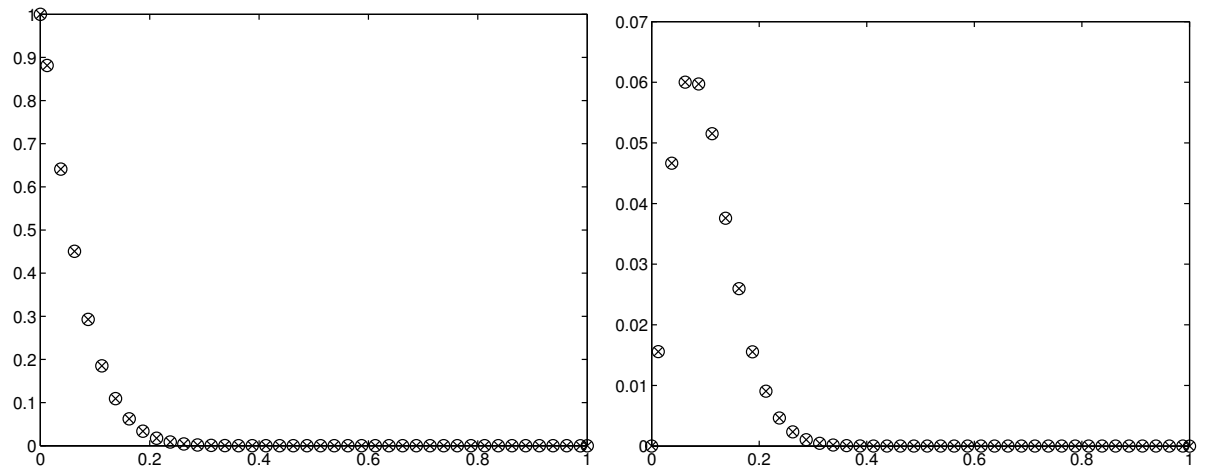

FIG. 8.18. The linear transport equation in high dimension $d=10$ : The mean (left) and standard deviation (right) of $\bar{r}$ at $\epsilon=10^{-8}$, obtained by $3 r d$-order gPC Galerkin (circles) and the level-2 sparse grids stochastic collocation method (crosses).

9. Summary. In this paper, we proposed a class of stochastic asymptotic preserving (sAP) schemes for multiscale kinetic and hyperbolic equations with diffusive scalings. The new schemes are built upon the deterministic AP schemes. They employ the generalized polynomial chaos (gPC) expansion, combined with stochastic Galerkin projection. We show that the new schemes are AP in the stochastic setting. We also discussed the use of stochastic Galerkin and stochastic collocation. We made it clear that the Galerkin approach is preferred as it guarantees the AP property in the entire random space, regardless of the order of the approximation. On the other hand, if one is only interested in the solution statistics (e.g., mean, variance, etc), then stochastic collocation is easier to implement in practice. This paper serves as the first attempt to develop AP schemes in stochastic setting, and we intend to study more in-depth issues in future studies. 
Acknowledgments. S. Jin was supported by National Science Foundation of China grant No. 91330203, and the NSF DMS grants No. 1107291 and no. 1107291: RNMS "KI-Net". D. Xiu was partially supported by AFOSR and DOE.

\section{REFERENCES}

[1] C. Bardos, R. Santos, and R. Sentis. Diffusion approximation and computation of the critical size. Trans. Amer. Math. Soc., 284(2):617-649, 1984.

[2] Russel E. Caflisch, Shi Jin, and Giovanni Russo. Uniformly accurate schemes for hyperbolic systems with relaxation. SIAM J. Numer. Anal., 34(1):246-281, 1997.

[3] T. Carleman. Problèmes mathématiques dans la théorie cinétique des gaz. Publ. Sci. Inst. Mittag-Leffler. 2. Almqvist \& Wiksells Boktryckeri Ab, Uppsala, 1957.

[4] Kenneth M. Case and Paul F. Zweifel. Linear transport theory. Addison-Wesley Publishing Co., Reading, Mass.-London-Don Mills, Ont., 1967.

[5] Yan Ding, Tiejun Li, Dongxiao Zhang, and Pingwen Zhang. Adaptive Stroud stochastic collocation method for flow in random porous media via Karhunen-Loève expansion. Commun. Comput. Phys., 4(1):102-123, 2008.

[6] Anil K. Prinja Erin D. Fichtl and James S. Warsa. Stochastic methods for uncertainty quantification in radiation transport. In International Conference on Mathematics, Computational Methods 86 Reactor Physics (MEC 2009), Saratoga Springs, New York, May 3-7, 2009, 2009.

[7] Francis Filbet and Shi Jin. A class of asymptotic-preserving schemes for kinetic equations and related problems with stiff sources. Journal of Computational Physics, 229(20):7625-7648, 2010.

[8] B. Despres G. Poette and D. Lucor. Uncertainty quantification for systems of conservation laws. J. Comput. Phys., 228(7):2443-2467, 2009.

[9] R.G. Ghanem and P. Spanos. Stochastic Finite Elements: a Spectral Approach. SpringerVerlag, 1991.

[10] S. Goldstein. On diffusion by discontinuous movements, and on the telegraph equation. Quart. J. Mech. Appl. Math., 4:129-156, 1951.

[11] François Golse, Shi Jin, and C. David Levermore. The convergence of numerical transfer schemes in diffusive regimes. I. Discrete-ordinate method. SIAM J. Numer. Anal., 36(5):1333-1369, 1999.

[12] Laurent Gosse and Giuseppe Toscani. Space localization and well-balanced schemes for discrete kinetic models in diffusive regimes. SIAM J. Numer. Anal., 41(2):641-658 (electronic), 2003.

[13] D. Gottlieb and D. Xiu. Galerkin method for wave equations with uncertain coefficients. Comm. Comput. Phys., 3(2):505-518, 2008.

[14] M. Ndjinga J. Tryoen, O. Le Maitre and A. Ern. Intrusive galerkin methods with upwinding for unceraintynonlinear hyperbolic systems. J. Comput. Phys., 228(7):2443-2467, 2009.

[15] S. Jin. Asymptotic preserving (ap) schemes for multiscale kinetic and hyperbolic equations: a review. Lecture Notes for Summer School on Methods and Models of Kinetic Theory(MEMKT), Porto Ercole (Grosseto, Italy), 2010.

[16] S. Jin, L. Pareschi, and G. Toscani. Diffusive relaxation schemes for multiscale discrete-velocity kinetic equations. SIAM Journal on Numerical Analysis, 35(6):2405-2439, 1998.

[17] S. Jin, L. Pareschi, and G. Toscani. Uniformly accurate diffusive relaxation schemes for multiscale transport equations. SIAM Journal on Numerical Analysis, 38(3):913-936, 2000.

[18] Shi Jin. Runge-Kutta methods for hyperbolic conservation laws with stiff relaxation terms. $J$. Comput. Phys., 122(1):51-67, 1995.

[19] Shi Jin. Efficient asymptotic-preserving (AP) schemes for some multiscale kinetic equations. SIAM J. Sci. Comput., 21(2):441-454 (electronic), 1999.

[20] Shi Jin and C. David Levermore. Numerical schemes for hyperbolic conservation laws with stiff relaxation terms. J. Comput. Phys., 126(2):449-467, 1996.

[21] Shi Jin and Zhouping Xin. The relaxation schemes for systems of conservation laws in arbitrary space dimensions. Communications on pure and applied mathematics, 48(3):235-276, 1995.

[22] Axel Klar. An asymptotic-induced scheme for nonstationary transport equations in the diffusive limit. SIAM J. Numer. Anal., 35(3):1073-1094 (electronic), 1998.

[23] Edward W. Larsen and Joseph B. Keller. Asymptotic solution of neutron transport problems for small mean free paths. J. Mathematical Phys., 15:75-81, 1974.

[24] Edward W. Larsen and J. E. Morel. Asymptotic solutions of numerical transport problems in optically thick, diffusive regimes. II. J. Comput. Phys., 83(1):212-236, 1989. 
[25] Edward W. Larsen, J. E. Morel, and Warren F. Miller, Jr. Asymptotic solutions of numerical transport problems in optically thick, diffusive regimes. J. Comput. Phys., 69(2):283-324, 1987.

[26] Mohammed Lemou and Luc Mieussens. A new asymptotic preserving scheme based on micromacro formulation for linear kinetic equations in the diffusion limit. SIAM J. Sci. Comput., 31(1):334-368, 2008.

[27] Pierre Louis Lions and Giuseppe Toscani. Diffusive limit for finite velocity Boltzmann kinetic models. Rev. Mat. Iberoamericana, 13(3):473-513, 1997.

[28] Lorenzo Pareschi and Giovanni Russo. Implicit-Explicit Runge-Kutta schemes and applications to hyperbolic systems with relaxation. J. Sci. Comput., 25(1-2):129-155, 2005.

[29] Ada Pulvirenti and Giuseppe Toscani. Fast diffusion as a limit of a two-velocity kinetic model. In Proceedings of the VIII International Conference on Waves and Stability in Continuous Media, Part II (Palermo, 1995), number 45, part II, pages 521-528, 1996.

[30] T. Tang and T. Zhou. Convergence analysis for stochastic collocation methods to scalar hyperbolic equations with a random wave speed. Comm. Comp. Phys., 8:226-248, 2010.

[31] G.I. Taylor. Diffusion by continuous movements. Proc. London Math. Soc., 20:196-212, 1921.

[32] D. Xiu. Fast numerical methods for stochastic computations: a review. Comm. Comput. Phys., 5:242-272, 2009 .

[33] D. Xiu. Numerical methods for stochastic computations. Princeton Univeristy Press, Princeton, New Jersey, 2010.

[34] D. Xiu and J.S. Hesthaven. High-order collocation methods for differential equations with random inputs. SIAM J. Sci. Comput., 27(3):1118-1139, 2005.

[35] D. Xiu and G.E. Karniadakis. The Wiener-Askey polynomial chaos for stochastic differential equations. SIAM J. Sci. Comput., 24(2):619-644, 2002.

[36] D. Xiu and J. Shen. Efficient stochastic Galerkin methods for random diffusion equations. J. Comput. Phys., 228:266-281, 2009. 